\section{Fiber loop ringdown DNA and bacteria sensors}

\author{
Chamini Herath, ${ }^{\mathrm{a}}$ Chuji Wang, ${ }^{\mathrm{a}}$ Malik Kaya, ${ }^{\mathrm{a}}$ and David \\ Chevalierb $^{\text {b }}$ \\ a Mississippi State University, Department of Physics and Astronomy \\ and the Energy Institute, Mississippi State, Mississippi 39762 \\ ${ }^{b}$ Mississippi State University, Department of Biological Sciences, \\ Mississippi State, Mississippi 39762
}

\begin{abstract}
We report a new type of refractive index-based biosensor using a fiber loop ringdown evanescent field (FLRD-EF) sensing scheme, in which the sensing signal is a time constant and detection sensitivity is enhanced by the multipass nature of the ringdown technique. Bulk index-based detections of three different single strand DNAs and one type of bacteria are demonstrated for the FLRD-EF sensors that utilize a partially-etched single mode fiber as the sensor head. Stepwise coating of the sensor head with poly-L-lysine and a probe DNA has enabled surface index-based label-free target DNA sensing. We expect an array of FLRD-EF biosensors to be created, which are superior to counterparts in terms of simplicity, low cost, and high sensitivity. $\odot 2011$ Society of Photo-Optical Instrumentation Engineers (SPIE). [DOI: 10.1117/1.3572046]
\end{abstract}

Keywords: biosensors; fiber loop ringdown; evanescent field, DNA; bacteria.

Paper 11046LR received Feb. 1, 2011; revised manuscript received Mar. 5, 2011; accepted for publication Mar. 9, 2011; published online May 2, 2011.

Fiber loop ringdown (FLRD) is a relatively new sensing technique which has evolved from cavity ringdown spectroscopy. ${ }^{1}$ Since its first introduction for trace gas detection in $2002,{ }^{2}$ FLRD as a uniform time-domain sensing scheme has been explored to develop a variety of physical and chemical fiber optics sensors, such as pressure, strain, temperature, refractive index, microfluids, and volatile organic compounds; the details can been read in recent reviews. ${ }^{3,4}$ In FLRD, the sensing signal is a time constant; the detection sensitivity is proportionally enhanced by the number of round-trips a laser pulse travels in the fiber loop which can be up to a few kilometers long. Many sensing mechanisms, such as direct gas absorption, microbending-induced deformation, fiber Bragg grating (FBG)and long period grating (LPG)-based wavelength shift, and evanescent field (EF) absorption and scattering, can be directly adopted into the uniform sensing platform for the development of different sensors. This technique has great potential for biosensor development, yet it has not been much explored, except for one early publication in which a single mammalian cancer cell is detected based on the scattering effect of the localized EF around a $10-\mathrm{mm}$ long single mode fiber taper. ${ }^{5}$

In this work, we demonstrate bulk index-based deoxyribonucleic acid (DNA) and bacteria sensing and surface index-based

Address all correspondence to: Chuji Wang, Mississippi State University, Department of Physics and Astronomy, and the Energy Institute, Mississippi State, MS 39762. Tel: 662-325-9455; Fax: 662-325-8465; E-mail: cw175@msstate.edu. label-free DNA sensing using the FLRD sensing scheme combined with the EF sensing mechanism. To the best of our knowledge, this work presents the first DNA and bacteria sensors using the FLRD technique. Without utilizing additional optical components, such as an FBG or LPG to fabricate the sensor head, as reported in recent studies, ${ }^{6-10}$ our sensor design demonstrates comparable or better performance while featuring significantly lower cost, simplified design and configuration, and potentially higher detection sensitivity.

Figure 1(a) shows the fiber loop ringdown system consisting of a section of fused-silica single mode fiber (SMF 28, Corning, Inc.), two identical $2 \times 1$ fiber couplers (Opneti Communication), a temperature-controlled continuous wave diode laser with output power of $30 \mathrm{~mW}$ when operating at $100 \mathrm{~mA}$ (NEL America), an InGaAs photodetector (Thorlabs, PDA50B), and an electronic control. Cladding and core diameters of the single mode fiber are 125 and $8 \mu \mathrm{m}$, respectively. The total optical loss, including the absorption loss, fiber connectors' insertion losses, and fiber couplers' losses, was estimated to be $<0.45 \mathrm{~dB}$. The typical splicing loss, which was estimated by the splicer, was 0.02 to $0.04 \mathrm{~dB}$ and the total fiber scattering loss was negligible. The total length of the loop was $120 \mathrm{~m}$.

Once a laser beam was coupled into the fiber loop, the ringdown signal detected by the photodetector was applied to the pulse generator (SRS, DG 535) as an external trigger. A series of laser pulses were generated with a pulse duration of approximately $20 \mathrm{~ns}$. The photodiodes then detect a series of spikes whose envelope forms an exponential decay curve. The resultant ringdown time (the decay constant) was on the order of tens of $\mu$ microseonds. The amplification of the detector was set at $50 \mathrm{~dB}$ signal-to-noise ratio with the minimum detectable power of $10 \mathrm{nW}$.

The EF sensor heads, as shown in Fig. 1(b), were fabricated using the chemical etching method. The etching process was monitored in real-time, on-line by the FLRD technique. A 24-cm long section of the fiber was selected and the plastic outer jacket was removed. The bare section of fiber was immersed in a $48 \%$ hydrofluoric acid (HF) solution and the section of the fiber in contact with the acid was gradually etched away. When the EF propagating through the cladding started to leak out to the external medium, the observed ringdown time began to decrease due to the increase of the optical loss. A typical ringdown time change was 4 and $5 \mu \mathrm{s}(\sim 0.05 \mathrm{~dB}$ loss $)$ before and after etching process, depending on individual sensor units. The diameter of the etched fiber was estimated from the recorded ringdown time and further verified by using scanning electron microscopy. The etched fiber diameter was controlled at approximately $10 \mu \mathrm{m}$ after 32 or 33 min etching process.

The response of the above sensor head to the biological sample solutions containing DNA and bacteria was investigated. Three different DNA samples (Integrated DNA Technologies) were used in this experiment. Sample 1 (S1) was 26 base pairs single strand DNA (ssDNA) with the sequence CAG CGA GGT GAA AAC GAC AAA AGG GG, Sample 2 (S2) was another 26 base pairs ssDNA with the sequence CCC CTT TTG TCG TTT TCA CCT CGC TG. The other DNA sample (S3) was a combination of $\mathrm{S} 1$ and $\mathrm{S} 2,26$ base pairs double strand DNA (dsDNA).

1083-3668/2011/16(5)/050501/3/\$25.00 @ 2011 SPIE 


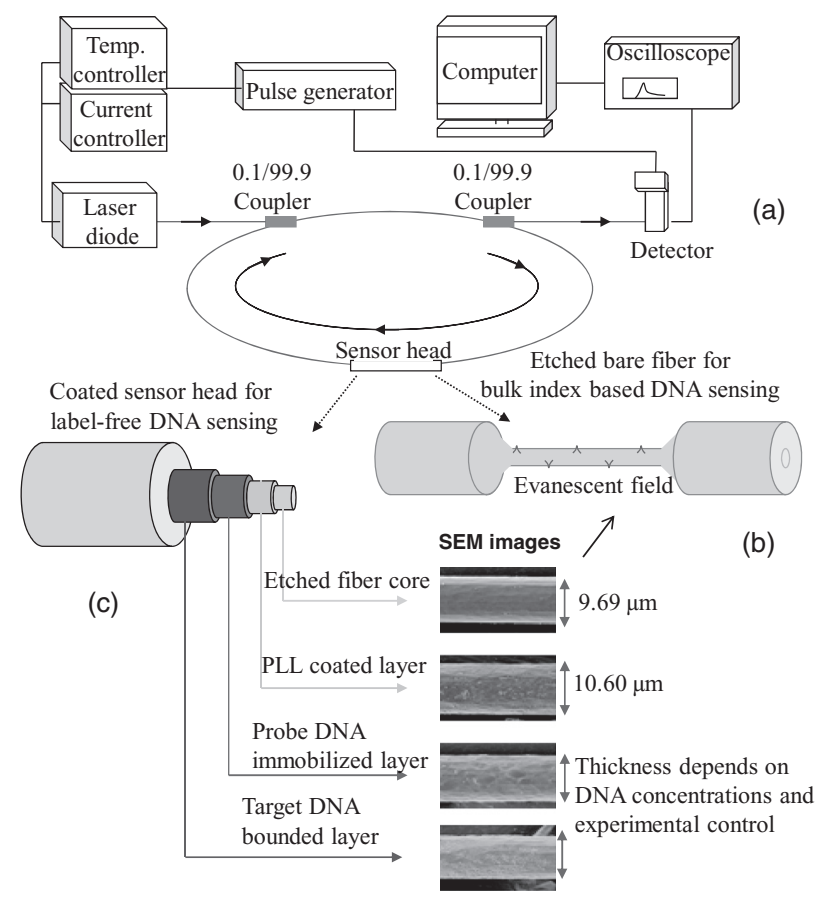

Fig. 1 (a) Schematic of the FLRD-EF sensor, (b) The etched bare single mode fiber sensor head for bulk index-based sensing, (c) The coated sensor head for label-free DNA sensing.

Figures 2(a) and 2(b) show the typical response of the sensor head to the external media: air, water, S1, S2, and S3. In the first part of the experiment, the DNA sample was diluted in deionized (DI) water to $30 \mu \mathrm{M}$ to be used as the sample solution [Fig. 2(a)] and then the DNA solution was further diluted to 15 $\mu \mathrm{M}$ [Fig. 2(b) ] in order to examine the concentration detection limit. Both experiments were performed at room temperature $\left(22^{\circ} \mathrm{C}\right)$ and the wavelength of the diode laser was maintained at $1515.15 \mathrm{~nm}$, where water and atmospheric constituents have negligible absorption (the absorption is too weak to be detected by the current sensor). As shown in Fig. 2, the response to the change in the external media is fast $(<1 \mathrm{~s})$ and reproducible. Different refractive indices (RIs) of the media affect the EF scattering effect: ${ }^{11}$ thus, generating different optical losses, which are observed by a change in ringdown time. This result indicates that given the same concentration of the DNA solutions, the different DNAs that have different bulk RIs can be discriminated by the sensor. The minimum detectable RI difference in different sample solutions was estimated to be as small as $0.00003 \mathrm{RIU}$, which was not detectable by conventional single-pass EF sensors using a bare single mode fiber as the sensor head. ${ }^{12}$ Based on the index-difference $(\Delta n)$ sensing mechanism, a smaller $\Delta n$ $\left(n_{\text {fibercore }}-n_{\text {medium }}\right)$ corresponds to a lower optical loss (a longer ringdown time), ${ }^{11,12}$ e.g., $\Delta n_{\text {(fiber core - water) }}<\Delta n_{\text {(fiber core - air) }}$. The different ringdown times at S1, S2, and S3 indicate that $\Delta n_{\text {(S1-air) }}<\Delta n_{\text {(S3-air) }}<\Delta n_{\text {(S2-air) }}$ while the actual RIs were not calibrated.

The RI-based sensing was further investigated by testing DI water and bacteria (Escherichia coli strain $\mathrm{DH} 5 \alpha$ ) that was suspended in DI water. The size of the bacteria was $\sim 2 \mu \mathrm{m}$ long and $0.5 \mu \mathrm{m}$ in diameter, which was significantly larger than DNA. The concentration of the bacteria was $1.4 \times 10^{9} \mathrm{cell} / \mathrm{mL}$.
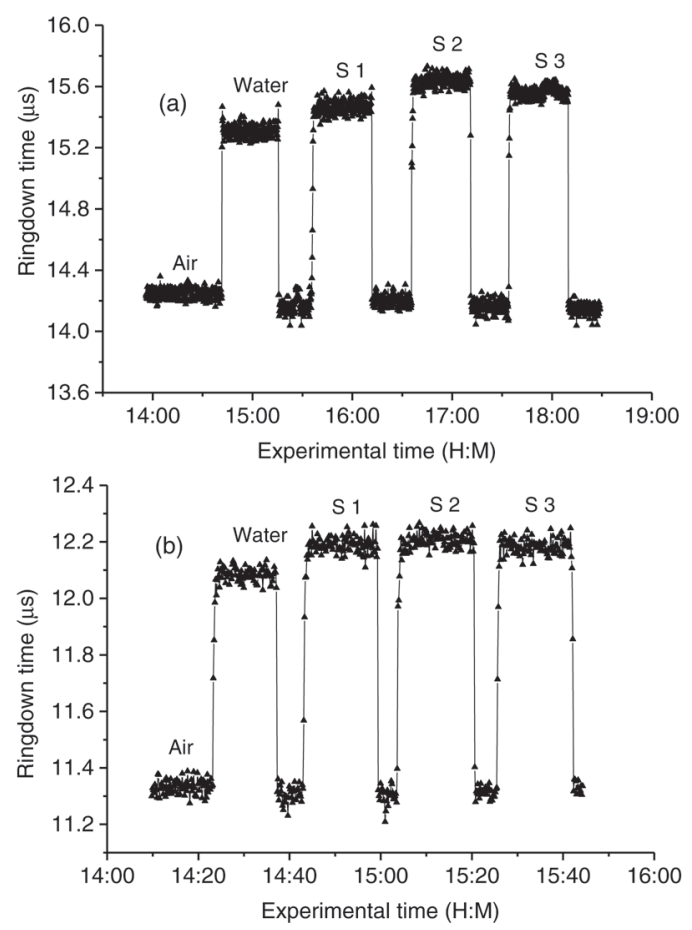

Fig. 2 Demonstration of the FLRD-EF sensor for the bulk index-based detections of ssDNA sample 1 (S1), ssDNA sample 2 (S2), and dsDNA sample 3 (S3). (a) DNA concentration $30 \mu \mathrm{m}$ and (b) DNA concentration $15 \mu \mathrm{m}$.

As shown in Fig. 3, the sensor can readily discriminate the bacteria solution from DI water. The sensor showed a fast and reversible response to the change of samples.

Using a coating method similar to the technique reported by Jang et al. ${ }^{6}$ and Chen et al., ${ }^{7}$ we investigated the feasibility of label-free DNA sensing by the sensor. The partially-etched fiber was first cleaned with phosphate-buffered saline solution $\left(\mathrm{NaH}_{2} \mathrm{PO}_{4} / \mathrm{Na}_{2} \mathrm{HPO}_{4} p \mathrm{H} 7.4,150 \mathrm{mM}\right)(\mathrm{PBS})$. In order to be effectively coated with poly-L-lysine (PLL) solution $(0.1 \% \mathrm{~W} / \mathrm{V}$ in water, the molecular weight $=150,000$ to $300,000 \mathrm{~g} / \mathrm{mol}$ ), the sensor head was immersed in PLL for 160 min. The sensor head was then cleaned with PBS to remove excess PLL and immersed for $130 \mathrm{~min}$ in $20 \mu \mathrm{m}$ ssDNA S1. Again cleaned with PBS, the sensor head, which was then coated with S1 (the probe DNA), was ready for sensing the matched S2 (the target DNA). The entire stepwise coating process, illustrated in

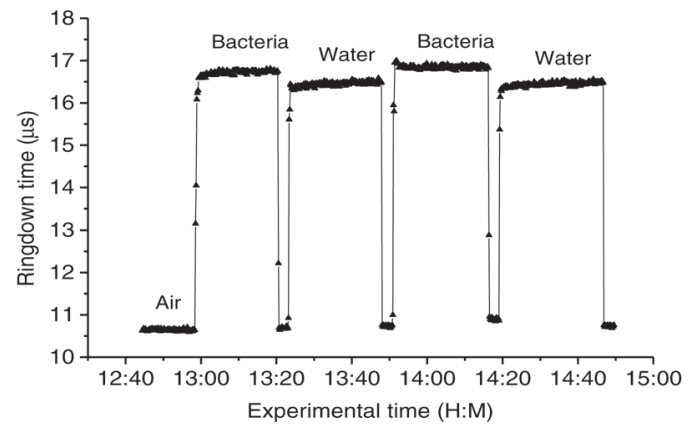

Fig. 3 Demonstration of the FLRD-EF sensor for the detection of bacteria. 


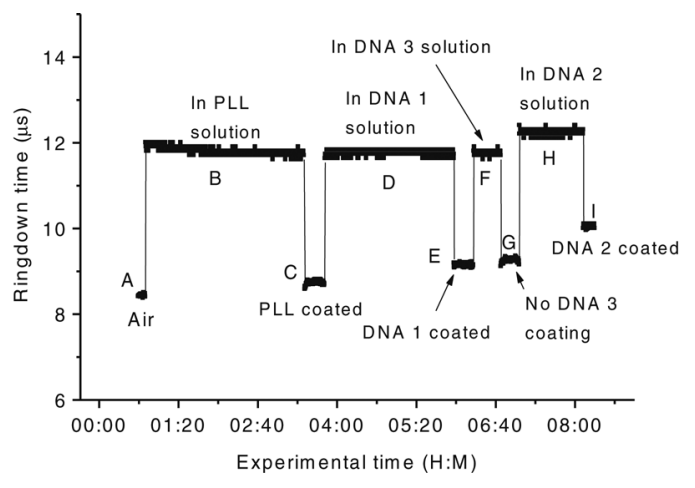

Fig. 4 Surface index-based label-free DNA sensing of the FLRD-EF sensor. The probe DNA (S1) immobilized sensor head selectively senses the target DNA (S2) but it has no response to the non-matched DNA (S3).

Fig. 1(c), was performed at room temperature and the ringdown data was continuously collected throughout the experiment. Since the positively charged $\mathrm{NH}_{3}{ }^{+}$in the PLL chain is binding to the negatively charged DNA, and the probe DNA (S1) selectively binds with the target DNA (S2), the potential label-free DNA sensing should be achievable by the new sensor described in this work. In the experiment, before the sensor head was used to sense the target DNA, the sensor head was immersed in the non-matched DNA S3 with a total concentration $20 \mu \mathrm{Mm}(\mathrm{S} 1+\mathrm{S} 2)$ for $65 \mathrm{~min}$; S3 solution was then removed and the sensor head was cleaned with PBS. Next, the same sensor head was immersed in $20 \mu \mathrm{M}$ target DNA for $65 \mathrm{~min}$ and then cleaned with PBS. Figure 4 presents the measured ringdown times in each stage of the coating process labeled as A, B, C, D, E, F, G, H, and I, which denote prior-coating (inair), in-PLL-solution, PLL-coated, in-S1 solution, S1-coated, in-S3 solution, S3-coated, in-S2 solution, and S2-coated, respectively. The different coating effects in terms of changes in RI are clearly depicted by the different ringdown times observed.

The selective response of the sensor to DNA samples S2 and $\mathrm{S} 3$ can be seen in Fig. 4. The sensor shows the same ringdown time, $9.2 \mu \mathrm{s}$, in stages $\mathrm{E}$ and G. However, when the $\mathrm{S} 1$ coated sensor head was immersed in the S2 solution the sensor head was successfully coated with $\mathrm{S} 2$ and the observed ringdown time was $10.0 \mu \mathrm{s}$, as shown by stage I. This selective response is due to the chemically selective binding of S2 to $\mathrm{S} 1$ and the non-match behavior between S3 and S1. This result indicates that the immobilization of a probe DNA (S1) on the PLL coated sensor head can selectively detect a target DNA (S2).

The detection sensitivity of the sensor is affected by several factors, including the etched fiber diameter the length of the sensor head and the physical parameters of the fiber loop. For a given fiber loop ringdown sensor unit a thin coating of a specific chemical on the etched fiber not only achieves selectivity but also enhances sensitivity. As shown in Fig. 4, the difference in ringdown time between the two stages D (in-S1) and H (in-S2) was $0.49 \mu$ s, which was approximately 16 times larger than the recorded ringdown time difference in the non-coated sensor, $0.03 \mu \mathrm{s}$, as shown in Fig. 2(b). Given that the concentration of the DNA used in the coated fiber experiment $(20 \mu \mathrm{m})$ was higher than the previous one $(15 \mu \mathrm{m})$ used in the noncoated sensor head, the enhanced effect of the coating on the detection sensitivity was approximately 12-fold, which yielded a detection sensitivity for DNA solutions of $1.3 \mu \mathrm{m}$. This detection limit is comparable to the detection limits achieved in the LPG-based DNA sensors. 6,7

In conclusion, we have demonstrated a new type of biosensor based on the FLRD technique. The bulk RI-based detections of three DNAs and one type of bacteria and the surface RI-based label-free detection of DNA are successfully demonstrated. We expect an array of low cost, near real-time response, and high sensitivity fiber optic biosensors using the FLRD technique to be created.

This work was supported by the National Science Foundation through Grant No. CMMI-0927539.

\section{References}

1. G. Berden and R. Englen, Cavity Ring-Down Spectroscopy: Techniques and Applications, Wiley-Blackwell Publishing Ltd., West Sussex, UK (2009).

2. K. Atherton, G. Stewart, and B. Culshaw, "Gas detection by cavity ring-down absorption with a fibre optic amplifier loop," Proc. SPIE Int. Soc. Opt. Eng. 4577, 25-31 (2002).

3. H. Waechter, J. Litman, A.H. Cheung, J.A. Barnes, and H.P. Loock, "Chemical sensing using fiber cavity ring-down spectroscopy," Sensors 10, 1716-1742 (2010).

4. C. Wang, "Fiber loop ringdown-a time-domain sensing technique for multi-function fiber optic sensor platforms: current status and design perspectives," Sensors 9, 7595-7621 (2009).

5. P. B. Tarsa, A. D. Wist, P. Rabinowitz, and K. K. Lehmann, "Singlecell detection by cavity ring-down spectroscopy," Appl. Phys. Lett. 85, 4523-4525 (2004).

6. H. S. Jang, K. N. Park, J. P. Kim, S. J. Sim, O. J. Kwon, Y. G. Han, and K. S. Lee, "Sensitive DNA biosensor based on a long-period grating formed on the side-polished fiber surface," Opt. Express 17, 3855-3860 (2009).

7. X. Chen, L. Zhang, K. Zhou, E. Davies, K. Sugden, I. Bennion, M. Hughes, and A. Hine, "Real time detection of DNA interactions with long period fiber grating based biosensor," Opt. Lett. 32, 2541-2543 (2007).

8. A. N. Chryssis, S. S. Saini, S. M. Lee, H. Yi, W. E. Bentley, and M. Dagenais, "Detecting hybridization of DNA by highly sensitive evanescent field etched core fiber bragg grating sensors," IEEE 11, 864-872 (2005).

9. S. Maguis, G. Laffont, P. Ferdinand, B. Carbonnier, K. Kham, T. Mekhalif, and M. Millot, biofunctionalized tilted fiber bragg gratings for label-free immunosensing, Opt. Express 16, 19049-19062 (2008).

10. J. L. Tang, S. F. Cheng, W. T. Hsu, T. Y. Chiang, and L. K. Chau, "Fiber optic biochemical sensing with a colloidal gold modified long period fiber grating," Sensors and Actuators B 119, 105-109 (2006).

11. P. Polynkin, A. Polynkin, N. Peyghambarian, and M. Mansuripur, "Evanescent field-based optical fiber sensing device for measuring the refractive index of liquids in microfluidic channels," Opt. Lett. 30, 1273-1275 (2005).

12. C. Wang and C. Herath, "High-sensitivity fiber-loop ringdown evanescent-field index sensors using single-mode fiber," Opt. Lett. 35, 1629-1631 (2010). 